Keiko Wakui • Mitsuyo Tanemura $\cdot$ Kaoru Suzumori

Eiko Hidaka • Masayo Ishikawa • Takeo Kubota

Yoshimitsu Fukushima

\title{
Clinical applications of two-color telomeric fluorescence in situ hybridization for prenatal diagnosis: Identification of chromosomal translocation in five families with recurrent miscarriages or a child with multiple congenital anomalies
}

Received: September 9, 1998 / Accepted: October 23, 1998

\begin{abstract}
Two-color fluorescence in situ hybridization (FISH) analysis using human chromosome arm-specific telomeric probes (telomeric probes) was used successfully to detect each derivative chromosome of a translocation carrier in five couples who requested a prenatal diagnosis in future pregnancies. Most of the human chromosome terminal bands are G-band-negative, and even FISH analysis using whole-chromosome painting (wcp) probes are often of insufficient complexity to detect subtle chromosomal changes. A complete set of human telomeric probes was developed to improve the sensitivity of diagnosis of microdeletions or other cryptic rearrangements in telomeric regions. Two-color telomeric FISH was the only possible method for precise prenatal diagnosis of one of the couples, because the carrier's chromosomal aberration was too subtle to be detected by wcp FISH or conventional methods. We have demonstrated that two-color telomeric FISH has the potential to be a powerful new tool in the detection of cryptic chromosomal rearrangements involving telomeric regions in prenatal diagnosis precisely and in time.
\end{abstract}

Key words Chromosome rearrangement $\cdot$ Cryptic translocation · Fluorescence in situ hybridization (FISH) · Twocolor FISH · Telomeric probe · Whole-chromosome painting probe $\cdot$ Prenatal diagnosis $\cdot$ Genetic counseling

K. Wakui $\cdot$ T. Kubota $\cdot$ Y. Fukushima $(\square)$

Department of Hygiene and Medical Genetics, Shinshu University

School of Medicine, 3-1-1 Asahi, Matsumoto, Nagano 390-8621,

Japan

Tel. +81-263-37-2617; Fax +81-263-37-2619

e-mail: yfukush@sch.md.shinshu-u.ac.jp

M. Tanemura $\cdot$ K. Suzumori

Department of Obstetrics and Gynecology, Nagoya City University

Medical School, Aichi, Japan

E. Hidaka $\cdot$ M. Ishikawa

Department of Laboratory Medicine, Shinshu University School of

Medicine, Nagano, Japan

\section{Introduction}

The advances in molecular cytogenetics, especially the technique of fluorescence in situ hybridization (FISH), have allowed more precise investigation of chromosomal structure and function (Ledbetter 1992; Ohashi et al. 1994; Wakui et al. 1996; Speicher and Ward 1996; Lichter 1997; Suzumori et al. 1998). Conventional cytogenetic analyses can reveal many chromosome aberrations, but it is sometimes difficult to identify supernumerary marker chromosomes and small de novo unbalanced rearrangements. Centromeric satellite DNA probes and whole-chromosome painting (wcp) probes, which are specific for most individual chromosomes, have been used to analyze chromosomal regions which cannot be identified by conventional methods (Trask 1991). However, it is still difficult to identify telomeric regions in chromosomes by either conventional $\mathrm{G}$ banding or FISH with wcp probes, because most of the human chromosome terminal bands are G-bandnegative, and the wcp probes are often of insufficient complexity to detect subtle chromosomal changes, especially in the telomeric regions.

Recently, a complete set of human chromosome armspecific telomeric probes has been developed to provide new region-specific unique sequence DNA FISH probes (Ning et al. 1996). FISH using these arm-specific telomeric probes (telomeric FISH) would seem to be a sensitive method for diagnosis of cryptic or subtle chromosome aberrations, including those in telomeric regions (Wakui et al. 1997, 1998). Telomeric FISH would be especially useful in the prenatal diagnosis of an individual with such a subtle balanced translocation, because it is sometimes difficult to make a good preparation for karyotyping from cultured amniotic cells or chorionic villi.

Therefore, we used two-color telomeric FISH to investigate five families, and demonstrate its usefulness in making a prenatal diagnosis. 


\section{Materials and methods}

\section{Case descriptions}

Five couples who had experienced multiple miscarriages or had a live-born/stillborn infant with unbalanced chromosome translocations were investigated. The chromosome analyses revealed that one member of each couple had a balanced translocation involving the telomeric regions. The couples requested prenatal diagnoses in their future pregnancies.

Family 1. The first baby of this couple suffered from holoprosencephaly, and died $1 \mathrm{~h}$ after birth. The karyotype of the patient was 46, XX, add (7)(q36). Chromosome analyses of the parents revealed that the father had a balanced translocation (46, XY, t $(7 ; 16)(\mathrm{q} 36 ; \mathrm{q} 22)$ (Fig. $1 \mathrm{~A}(\mathrm{a})))$. Thus, the karyotype of the first baby was interpreted as 46, XX, der (7) t $(7 ; 16)(\mathrm{q} 36 ; \mathrm{q} 22)$ pat. The parents requested prenatal diagnosis in their second pregnancy. An amniotic fluid specimen was submitted for cytogenetic analysis at 15 weeks of gestation.

We labeled the 7qter probe (3K23/PAC) with dig-11dUTP and the 16qter probe (D3b1/cosmid) with bio-16dUTP for telomeric FISH analyses.

Family 2. The first child was affected with multiple congenital anomalies (MCAs), including hydrocephaly and mental retardation (MR). Chromosome analysis revealed that she had an additional chromosome segment on 4q. As her mother had a balanced translocation between $4 \mathrm{q}$ and $7 \mathrm{p}(46, \mathrm{XX}, \mathrm{t}(4 ; 7)(\mathrm{q} 35 ; \mathrm{p} 15.3))$, her chromosome aberration was $7 \mathrm{p}$ trisomy and $4 \mathrm{q}$ monosomy (46, XX, der (4) $\mathrm{t}(4 ; 7)(\mathrm{q} 35 ; \mathrm{p} 15.3)$ mat (Fig. 1B(a))). The parents requested prenatal diagnosis in the next pregnancy, and chorionic villus sampling was submitted for cytogenetic analysis at 10 weeks of gestation.

We labeled the 4qter probe (cT55/cosmid) with bio-16dUTP and the 7pter probe (109A6/cosmid) with dig-11dUTP for telomeric FISH analyses.

Family 3. The first child of this couple suffered from MCAs with suspicion of the cat cry syndrome. However, the initial conventional G-banding analysis failed to detect any abnormalities. FISH analysis using the chromosome 5p15. 2-specific probe (D5S23VYSIS) indicated that the child had a derivative chromosome 5 derived from an adjacent I segregation of the mother's subtle balanced translocation (46, XX.ish t(5; 10)(p15.1; p13)(D5S23-; D5S23+)(Fig. 1C(a))). Thus, the karyotype of the first child was interpreted as 46, XY. ish der (5) t (5; 10) (p15.1;p13)(D5S23-; D5S23+) mat. The derivative chromosomes 5 and 10 could not be detected by $\mathrm{G}$ banding because the segments involving the translocation are similar in size and in banding pattern. The couple requested prenatal diagnosis in their second pregnancy, and an amniotic fluid specimen was submitted for cytogenetic analysis at 15 weeks of gestation.

We labeled the 5pter probe (84C11/cosmid) with bio-16-
dUTP and the 10pter probe (2189b6/cosmid) with dig-11dUTP for telomeric FISH analyses.

Family 4. This couple had three miscarriages, and chromosome analyses revealed that the husband had a balanced translocation between $1 \mathrm{q}$ and $5 \mathrm{q}(46, \mathrm{XY}, \mathrm{t}(1 ; 5)(\mathrm{q} 42 ; \mathrm{q} 33)$ (Fig. 1D(a))). Telomeric FISH investigation was requested by the couple in future pregnancies.

We labeled the 1qter probe (112N326Q6/cosmid) with dig-11-dUTP and the 5qter probe (B22a4/cosmid) with bio16-dUTP for telomeric FISH analysis.

Family 5. This couple had a history of two stillborns with holoprosencephaly. The initial chromosome analyses of the second stillborn baby and of the couple failed to find any chromosome abnormalities. As one of the causes of holoprosencephaly was 7q deletion (Gurrieri et al. 1993), special attention was focused on the chromosome 7 pairs of the couple by high-resolution $\mathrm{G}$ banding. Finally, we found that the father had a subtle balanced translocation between $7 \mathrm{q}$ and $13 \mathrm{q}(46, \mathrm{XY}, \mathrm{t}(7 ; 13)(\mathrm{q} 36.2 ; \mathrm{q} 34)$ (Fig. 1E(a))). The couple wanted to know whether prenatal diagnosis was possible in future pregnancies.

We labeled the 7qter probe (3K23/PAC) with dig-11dUTP and the 13qter probe (85A10/P1) with bio-16-dUTP for telomeric FISH analysis.

Fig. 1A-E Partial karyotypes by GTG banding and ideograms of the carriers and fluorescence in situ hybridization (FISH) using telomeric probes and/or painting probes of the carrier and/or fetuses. In a partial karyotype in each case, an arrow indicates a breakpoint on the normal chromosome, and each arrowhead shows a rejoining point on the rearranged chromosome. In telomeric FISH in each case, a red arrow indicates signals from the digoxigenin-labeled probe, and a green arrow indicates signals from the biotin-labeled probe. A Family 1. A Gbanded partial karyotype of the father showing a balanced translocation $(\mathrm{t}(7 ; 16)(\mathrm{q} 36 ; \mathrm{q} 22))(a)$. FISH analysis using 7qter (red arrows) and $16 q$ ter probes (green arrows) of the father $(b)$ (the 16q telomeric probe cross-hybridizes weakly to 3qter, 9qter, 10pter, 11pter, and 19qter in this figure) and an amniotic fluid specimen $(c)$. B Family 2. A G-banded partial, karyotype of the mother showing a balanced translocation $(\mathrm{t}(4 ; 7)(\mathrm{q} 35 ; \mathrm{p} 15.3))(a)$. FISH analysis using 4qter (green arrows) and 7 pter probes (red arrows) of the mother $(b)$ and chorionic villus samples $(c)$. C Family 3. A G-banded partial karyotype of the mother showing a balanced translocation $(\mathrm{t}(5 ; 10)(\mathrm{p} 15.1 ; \mathrm{p} 13))(a)$. FISH analysis using 5 pter (green arrows) and 10pter probes (red arrows) of the mother $(b)$ and an amniotic fluid specimen (c). D Family 4. A G-banded partial karyotype showing a balanced translocation $(\mathrm{t}(1 ; 5)(\mathrm{q} 42 ; \mathrm{q} 33))(a)$, and FISH analysis using 1qter (red arrows) and 5 qter probes (green arrows) (b), of the husband. E Family 5. A Gbanded partial karyotype of the father showing a balanced translocation $(\mathrm{t}(7 ; 13)(\mathrm{q} 36.2 ; \mathrm{q} 34))(a)$. FISH analysis using 7qter (red arrows) and 13qter probes (green arrows) of the father. The signals of the 7qter and 13qter probes are detectable not only in the metaphase but also in the interphase $(b)$. FISH analysis using wcp 7 (VYSIS) (c) and wcp 13 (VYSIS) $(d)$ of the father. In wcp FISH, each target chromosome fluoresces red, and a red arrow indicates the small signals of wcp 7 on der(13). White arrows indicate normal and derivative chromosomes 7, and white arrowheads indicate normal and derivative chromosomes 13, respectively 


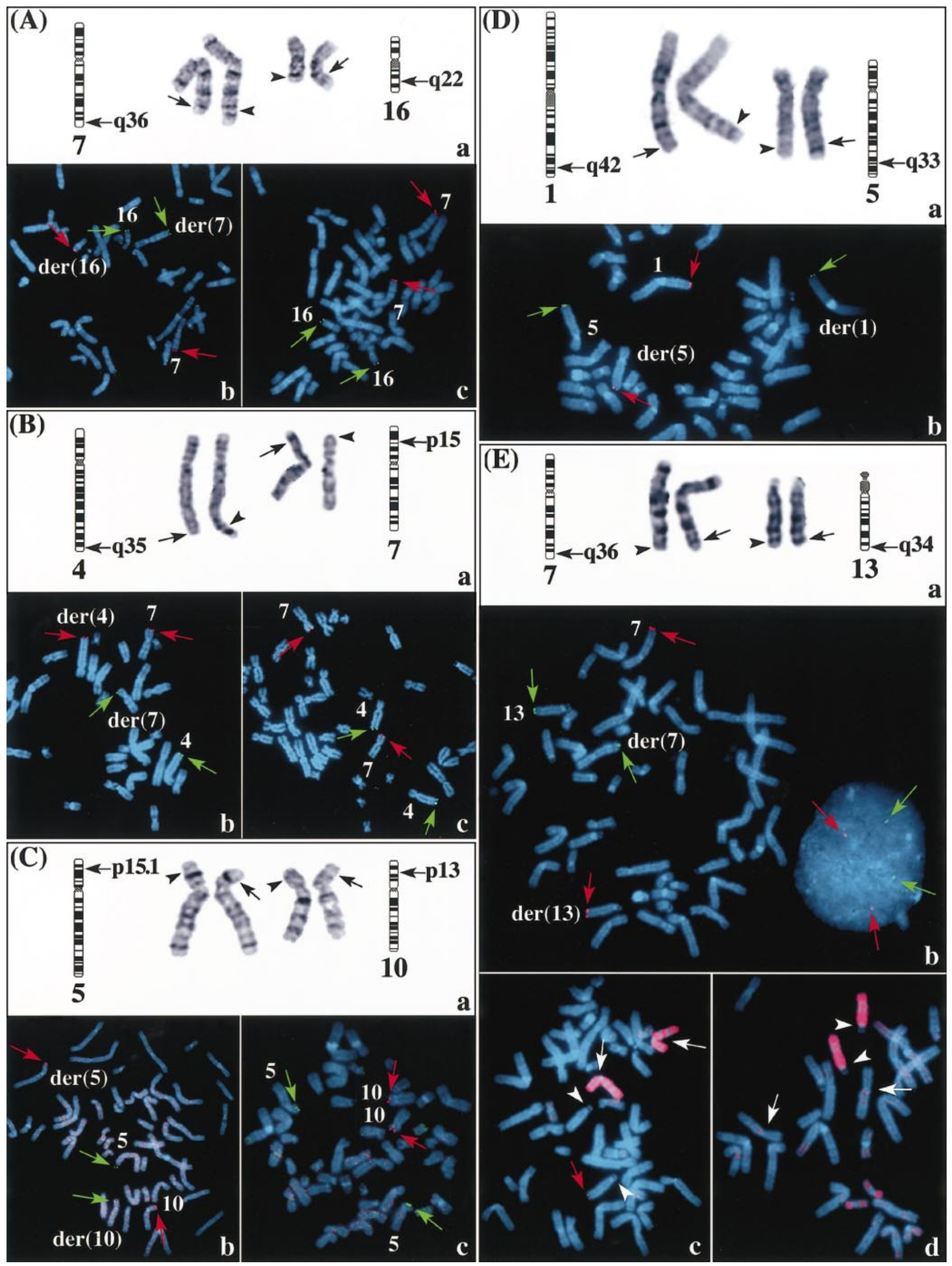


Two-color FISH analysis with telomeric probes

Metaphase chromosome preparations were made from peripheral blood, amniocytes or chorionic villus cells by standard methods.

The slides were incubated in a $2 \times$ saline - sodium citrate (SSC) solution at $37^{\circ} \mathrm{C}$ for $15 \mathrm{~min}$ followed by dehydration in ethanol, and were denatured in $70 \%$ formamide/ $2 \times \mathrm{SSC}$ at $75^{\circ} \mathrm{C}$ for $2 \mathrm{~min}$, immersed in $70 \%$ ethanol at $-20^{\circ} \mathrm{C}$, and dehydrated through an ethanol series again. For each case, two kinds of target chromosome arm-specific telomeric probes were used for two-color FISH analysis (Ning et al. 1996). Each probe DNA was labeled by nick translation with either biotin-16-dUTP or digoxigenin-11dUTP. The final concentration was 200-300ng for the cosmid clone DNA probe and $100 \mathrm{ng}$ for the $\mathrm{PAC} / \mathrm{P} 1$ clone DNA probe in $10 \mu l$ of the hybridization mixture per slide. After purification by the ethanol precipitation method, the biotinylated and digoxigeninylated probe DNAs were suspended in formamide solution. They were mixed using a biotin-labeled probe:digoxigenin-labeled probe ratio of $7: 3(\mathrm{v} / \mathrm{v})$, with an excess of Cot-1 DNA of about 5-50-fold, and were denatured (Inazawa et al. 1992). The denatured probes were mixed with hybridization solution and pipetted onto the denatured chromosome preparations. The preparations were incubated to hybridize the DNAs overnight at $37^{\circ} \mathrm{C}$. After being washed with $50 \%$ formamide $/ 2 \times \mathrm{SSC}$ at $37^{\circ} \mathrm{C}, 2 \times \mathrm{SSC}$ and $1 \times \mathrm{SSC}$ at room temperature for $15 \mathrm{~min}$ each, and $4 \times \mathrm{SSC}$ at room temperature for $5 \mathrm{~min}$, the slides were treated with the detection cocktail reagent, which included fluorescein isotheiocyanate - avidin and rhodamine antidigoxigenin, to detect biotin- and digoxigenin-labeled probes, respectively. Slides were mounted with a fluorescence antifade solution containing 4,6-diamidino-2-phenylindole (DAPI) and $p$ phenylenediamine.

We observed the signals from the chromosomes under an epifluorescence microscope (Olympus BX-50 Olympus, Tokyo, Japan) coupled to a charge-coupled device camera. Separate images of the DAPI chromosomes and the hybridization signals were merged using an image analysis system (CytoVision, Applied Imaging, Santa Clara, California, USA).

\section{Results}

\section{Family 1 (Fig. 1A)}

Two-color FISH analysis showed that the father had a balanced translocation (7qter signals were hybridized on a normal chromosome 7qter and a derivative chromosome $16 q$ ter, and 16qter signals were on a normal chromosome 16qter and a derivative chromosome 7qter) (Fig. 1A(b)); the first child had an unbalanced translocation with $7 \mathrm{q}$ monosomy and 16q trisomy (7qter signals were hybridized on only a normal chromosome 7qter, and 16qter signals were on two normal chromosomes 16qter and a derivative chromosome 7qter) (data not shown), and the fetus was karyotypically normal (7qter signals were hybridized on two normal chromosomes 7qter, and 16qter signals were on two normal chromosomes 16qter) (Fig. 1A(c)).

Family 2 (Fig. 1B)

Two-color FISH analysis of the mother showed that 4qter signals were on a normal chromosome 4qter and a derivative chromosome 7 pter, and 7 pter signals were hybridized on a normal chromosome 7pter and a derivative chromosome 4qter (Fig. 1B(b)). The fetus was considered to be karyotypically normal because 4qter signals were hybridized on two normal chromosomes 4qter, and 7pter signals were on two normal chromosomes 7pter (Fig. 1B(c)).

\section{Family 3 (Fig. 1C)}

Each derivative chromosome of the mother was easily detected by two-color FISH analysis (5pter signals were on a normal chromosome 5pter and a derivative chromosome 10 pter, and 10pter signals were hybridized on a normal chromosome 10pter and a derivative chromosome 5pter) (Fig. 1C(b)). The first child had an unbalanced translocation with $5 p$ monosomy and $10 p$ trisomy (5pter signals were on only a normal chromosome 5 pter, and 10pter signals were hybridized on two normal chromosomes 10pter and a derivative chromosome 5pter) (data not shown). The fetus was considered to have a normal karyotype (5pter signals were hybridized on two normal chromosomes 5pter, and 10 pter signals were on two normal chromosomes 10pter) (Fig. 1C(c)).

Family 4 (Fig. 1D)

Each derivative chromosome of the father was clearly detected (1qter signals were hybridized on a normal chromosome 1qter and a derivative chromosome 5qter, and 5qter signals were on a normal chromosome 5qter and a derivative chromosome 1qter (Fig. 1D(b)).

\section{Family 5 (Fig. 1E)}

Two-color FISH analysis showed that the father had a balanced translocation between $7 \mathrm{q}$ and $13 \mathrm{q}$ (7qter signals were hybridized on a normal chromosome 7qter and a derivative chromosome 13qter, and 13qter signals were on a normal chromosome 13qter and a derivative chromosome 7qter) (Fig. 1E(b)).

\section{Discussion}

A complete set of specific FISH probes representing each human telomere (telomeric probes) was developed to improve the sensitivity for detection of microdeletions or 
other cryptic rearrangements in the telomeric regions (Ning et al. 1996). Using these telomeric probes, we successfully detected each derivative chromosome of a translocation carrier in five couples who requested a prenatal diagnosis in future pregnancies. We describe in this study a practical application of telomeric FISH for prenatal diagnosis. In future prenatal diagnoses performed on a couple, one member of which has a reciprocal translocation, each derivative chromosome must be detected clearly. As a reciprocal translocation produces two derivative chromosomes, we performed two-color FISH analysis on the five translocation carriers. The two targeted telomeric probes were labeled simultaneously by biotin and digoxigenin. The telomeric regions of the two chromosomes involving the translocation could be successfully detected by this method. Using this technique, prenatal diagnosis was performed in the three couples (families 1 through 3 ), and revealed a normal karyotype in all cases.

As most of the terminal bands of human chromosomes are G-band-negative, subtle deletions or other structural aberrations in the telomeric regions are difficult to detect by $\mathrm{G}$ banding (Ning et al. 1996). Routine cytogenetic analysis has a 400-500 band resolution and is capable of detecting rearrangements involving 5-10-Mb DNA (Green et al. 1995). Even with high-resolution cytogenetic analysis, the limit of resolution is estimated to be $2-3 \mathrm{Mb}$, and larger rearrangements can potentially be overlooked if the translocated segments are shown by chromosome banding analysis to be similar in size and banding pattern. The use of wcp probes has have facilitated the detection of some subtle rearrangement. However, the telomeric regions are often under-represented, and the sensitivity of these probes for detecting subtle rearrangement is unclear. Thus, wcp probes may be inappropriate for the detection of subtle chromosome rearrangements in telomeric regions (Collins et al. 1991). The arm-specific telomeric probes within 100 $300 \mathrm{~kb}$ of the end of each chromosome arm increase the resolution of detection for telomeric regions by approximately 10-fold over current cytogenetic banding methods (Ning et al. 1996).

For instance, the initial chromosome analyses of the couple in family 5 indicated normal karyotypes. On the basis of the information on previous stillborns with holoprosencephaly from this family, high-resolution $G$ banding was able to reveal a subtle reciprocal translocation between $7 q$ and $13 q$ in the father (Fig. 1E(a)). However, the translocated segments were so small that each derivative chromosome is unlikely to be detected by conventional $\mathrm{G}$ banding in future prenatal diagnoses. Furthermore, we performed wcp FISH on this family, which revealed that the wcp probes failed in the detection of these derivative chromosomes. The derivative chromosome 13 could barely be visualized by FISH using wcp 7 in metaphases beyond the 400-band stage (Fig. 1E(c)), but below the 300-band stage (data not shown). The derivative chromosome 7 could not be detected by wcp 13 even in metaphases beyond the 400-band stage (Fig. 1E(d)). These results indicate that telomeric FISH is the only method that allows precise prenatal diagnosis in this family. Telomeric FISH is thus the most useful application in such cases.

Precise clinical diagnosis often gives us a clue to detect subtle chromosomal rearrangements in patients with MCAs/MR. In families 3 and 5, the particular chromosomal region investigated was based on the clinical diagnosis on the affected child and information on the locus of the causative gene. We were then able to make a precise cytogenetic diagnosis. Close cooperation between clinical geneticists and cytogenetisists is essential for high-quality cytogenetic investigation.

Although the reciprocal balanced translocations in families 1, 2 and 4 were identified by conventional cytogenetic techniques, we performed telomeric FISH analyses in order to obtain more definitive information for future prenatal diagnoses. It is sometimes difficult to obtain a suitable metaphase preparation from cultured amniotic fluid cells or chorionic villi. In such conditions, G-banding analysis is severely hampered, and it is difficult to detect each derivative or normal chromosome precisely (Suzumori et al. 1998). Moreover, it is possible for amniotic fluid cells or chorionic villi to grow poorly and not provide any metaphases. Two-color telomeric FISH supplies information not only on the target chromosomes of the metaphase but also on the nuclei of the interphase (Ohashi et al. 1993) (Fig. 1E(b)). Using telomeric FISH, we can easily detect each derivative or normal chromosome on even poorquality metaphases; moreover, we can ascertain whether the fetus is karyotypically balanced or not in the target chromosome regions on the basis of information on the numbers of each signal arising from the nuclei of the interphase. In the interphase diagnosis, we should be careful to use some FISH probes, such as the 16qter probes in family 1 , which produce weak cross-hybridization to a few other telomeres (Ning et al. 1996), although this cross-hybridization is negligible in the metaphase diagnosis.

Campana et al. (1986) reported that a reciprocal translocation carrier was found by conventional cytogenetics technique in $181 / 5445(3.3 \%)$ of the couples who had medical histories of recurrent abortions. On the other hand, Flint et al. (1995) reported that three in 99 idiopathic mentally retarded patients with normal routine karyotypes had subtelomeric chromosomal rearrangements using hypervariable DNA polymorphism analysis. Cryptic translocations and deletions involving telomeric regions seem not to be uncommon in couples with multiple miscarriages or in families of an unexplained MCA/MR child. Multicolor FISH is a new technique allowing the simultaneous visualization of multiple chromosomal targets in different colors. Very recently, the multicolor FISH protocol has been successfully applied to a set of armspecific telomeric probes (Lichter 1997). This approach will be helpful for the screening of couples with repeated spontaneous miscarriages and of unexplained MCA/MR patients.

The two-color FISH analysis with telomeric probes we describe here has the potential to be a powerful new tool, especially in prenatal diagnosis, for detecting cryptic chromosomal rearrangements involving telomeric regions. During the genetic counseling of couples with balanced 
translocations including the telomeric regions, more informed decisions concerning future pregnancies can be made by the use of telomeric FISH.

Acknowledgements We are grateful to Professor David H. Ledbetter and Dr. Christa M. Lese (The University of Chicago) for their kind provision of the telomeric probes used in this study, and for valuable suggestions. We also thank Dr. Makoto Kanai, Dr. Yasushi Takagi, Dr. Yukiko Hata, Dr. Seiichi Ushikubo, Dr. Tatsuya Yoda, Dr. Takashi Matsuoka (Shinshu University), and Dr. Naoki Niitsu (Niitsu Clinic) for providing clinical information of the patients, and Dr. Tsutomu Katsuyama (Shinshu University) for his useful advice. This work was supported in part by grants from the Ministry of Education, Science, Sports, and Culture, and from the Ministry of Health and Welfare, Japan.

\section{References}

Campana M, Serra A, Neri G (1986) Role of chromosome aberrations in recurrent abortion: a study of 269 balanced translocations. Am J Med Genet 24: 341-356

Collins C, Kuo WL, Segraves R, Fuscoe J, Pinkel D, Gray JW (1991) Construction and characterization of plasmid libraries enriched in sequences from single human chromosomes. Genomics 11: 997-1006

Flint J, Wilkie AOM, Buckle VJ, Winter RM, Holland AJ, McDermid HE (1995) The detection of subtelomeric chromosomal rearrangements in idiopathic mental retardation. Nat Genet 9: 132-139

Green ED, Cox DR, Myers RM (1995) The human genome progenct and its impact on the study of human disease. In: Scriver CR, Beaudet AL, Sly WS, Valle D (eds) The metabolic and molecular basis of inherited disease, 7th edn. McGraw-Hill, New York, pp 401-436

Gurrieri F, Trask BJ, van den Engh G, Krauss CM, Schinzel A, Pettenati MJ, Schindler D, Dietz-Band J, Vergnaud G, Scherer SW, Tsui LC, Muenke M (1993) Physical mapping of the holoprosencephaly critical region on chromosome 7q36. Nat Genet 3 : $247-251$
Inazawa J, Ariyama T, Abe T (1992) Physical ordering of three polymorphic DNA markers spanning the regions containing a tumor suppressor gene of renal cell carcinoma by three-color fluorescent in situ hybridization. Jpn J Cancer Res 83: 1248-1252

Ledbetter DH (1992) The "colorizing" of cytogenetics: is it ready for prime time? Hum Mol Genet 5: 297-299

Lichter P (1997) Multicolor FISHing: what's the catch? Trends Genet 13: 475-479

Ning Y, Roscke A, Smith ACM, Macha M, Precht K, Riethman H, Ledbetter DH, Flint J, Horsley S, Regan R, Kearney L, Knight S, Kvaloy K, Brown WRA (1996) A complete set of human telomeric probes and their clinical application. Nat Genet 14: 86-89

Ohashi H, Ishikiriyama S, Fukushima Y (1993) New diagnostic method for Pallister-Killian syndrome: detection of $i(12 p)$ in interphase nuclei of buccul mucosa by fluorescence in situ hybridization. Am J Med Genet 45: 123-128

Ohashi H, Wakui K, Ogawa K, Okano T, Niikawa N, Fukushima Y (1994) A stable acentric marker chromosome: possible existence of an intercalary ancient centromere at distal 8p. Am J Hum Genet 55: 1202-1208

Speicher MR, Ward DC (1996) The coloring of cytogenetics. Nat Med 2: $1046-1048$

Suzumori K, Tanemura M, Oya N, Suzumori N, Kim KC, Ohashi H, Fukushima Y (1998) Minute chromosomal rearrangements detected prenatally by fluorescence in situ hybridization. Prenat Disgn 18: $725-730$

Trask BJ (1991) Fluorescence in situ hybridization: applications in cytogenetics and gene mapping. Trends Genet 7: 149-154

Wakui K, Ohashi H, Yamagishi A, Hamada S, Nara T, Ishikiriyama S, Nakamura Y, Fukushima Y (1996) Interstitial duplication 8q22-q24: report of a case proven by FISH with mapped cosmid probes. Am J Med Genet 65: 36-39

Wakui K, Kubota T, Suzumori K, Ogata T, Ledbetter DH, Fukushima Y (1997) Advanced diagnosis of chromosome aberrations by FISH analysis with telomeric probes. Am J Hum Genet 61 (suppl): No. 810

Wakui K, Kubota T, Hidaka E, Ishikawa M, Katsuyama T, Suzumori K, Ohashi H, Ogata T, Yamamori S, Ledbetter DH, Fukushima Y (1998) Clinical applications of FISH analysis with human telomeric probes. J Hum Genet 43 (suppl): 55 (No. B-41) 